PREPARED FOR THE U.S. DEPARTMENT OF ENERGY, UNDER CONTRACT DE-AC02-76CH03073

PPPL-3829

PPPL-3829

UC-70

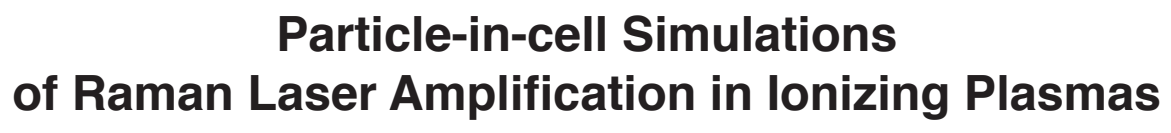

by

Daniel S. Clark and Nathaniel J. Fisch

June 2003

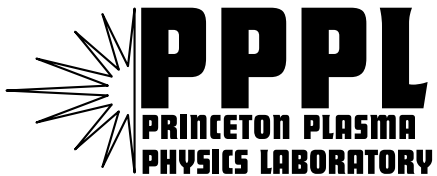

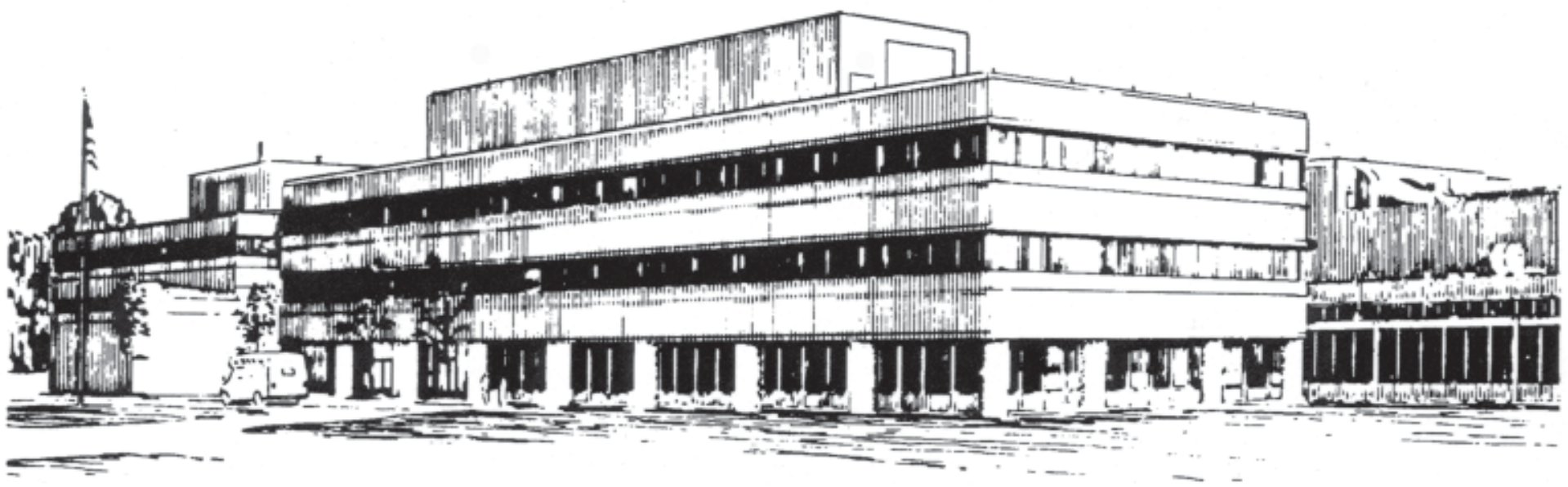

PRINCETON PLASMA PHYSICS LABORATORY PRINCETON UNIVERSITY, PRINCETON, NEW JERSEY 


\section{PPPL Reports Disclaimer}

This report was prepared as an account of work sponsored by an agency of the United States Government. Neither the United States Government nor any agency thereof, nor any of their employees, makes any warranty, express or implied, or assumes any legal liability or responsibility for the accuracy, completeness, or usefulness of any information, apparatus, product, or process disclosed, or represents that its use would not infringe privately owned rights. Reference herein to any specific commercial product, process, or service by trade name, trademark, manufacturer, or otherwise, does not necessarily constitute or imply its endorsement, recommendation, or favoring by the United States Government or any agency thereof. The views and opinions of authors expressed herein do not necessarily state or reflect those of the United States Government or any agency thereof.

\section{Availability}

This report is posted on the U.S. Department of Energy's Princeton Plasma Physics Laboratory Publications and Reports web site in Fiscal Year 2003. The home page for PPPL Reports and Publications is: http://www.pppl.gov/pub_report/

DOE and DOE Contractors can obtain copies of this report from:

U.S. Department of Energy

Office of Scientific and Technical Information

DOE Technical Information Services (DTIS)

P.O. Box 62

Oak Ridge, TN 37831

Telephone: (865) 576-8401

Fax: (865) 576-5728

Email: reports@adonis.osti.gov

This report is available to the general public from:

National Technical Information Service

U.S. Department of Commerce

5285 Port Royal Road

Springfield, VA 22161

Telephone: $1-800-553-6847$ or

(703) $605-6000$

Fax: (703) 321-8547

Internet: http://www.ntis.gov/ordering.htm 


\title{
Particle-in-cell simulations of Raman laser amplification in ionizing plasmas
}

\author{
Daniel S. Clark* and Nathaniel J. Fisch ${ }^{\dagger}$ \\ Plasma Physics Laboratory, Princeton University, P.O. Box 451, Princeton, New Jersey 08543
}

(Dated: June 27, 2003)

\begin{abstract}
By using the amplifying laser pulse in a plasma-based backward Raman laser amplifier to generate the plasma by photoionization of a gas simultaneous with the amplification process, possible instabilities of the pumping laser pulse can be avoided. Particle-in-cell simulations are used to study this amplification mechanism, and earlier results using more elementary models of the Raman interaction are verified [D.S.Clark and N.J.Fisch., Phys.Plasmas, 9(6):2772-2780, 2002]. The effects (unique to amplification in ionizing plasmas and not included in previous simulations) of blue-shifting of the pump and seed laser pulses and the generation of a wake are observed not significantly to impact the amplification process. As expected theoretically, the peak output intensity is found to be limited to $I \sim 10^{17} \mathrm{~W} / \mathrm{cm}^{2}$ by forward Raman scattering of the amplifying seed. The integrity of the ionization front of the seed pulse against the development of a possible transverse modulation instability is also demonstrated.
\end{abstract}

\section{INTRODUCTION}

A variation on the scheme of backward Raman laser pulse compression and amplification in plasma [1] has recently been proposed in the form of ionizing Raman amplification [2]. In the conventional Raman amplification scheme, a long pumping laser pulse is collided in a preformed plasma slab with a short seed pulse downshifted in frequency from the pump by the plasma frequency. The seed pulse serves to stimulate the Raman backscatter of the pump with the result that, in the nonlinear regime, the seed pulse is strongly amplified and also compressed temporally. Theoretically, unfocused intensities of $10^{17} \mathrm{~W} / \mathrm{cm}^{2}$ are accessible by this technique - an improvement of $10^{4-5}$ over current chirped pulse amplification techniques. In the ionizing Raman amplification scheme, in place of a preformed (i.e., ionized) plasma, a neutral precursor gas is used. Pump intensities below the photoionization threshold for that gas are then employed with a relatively more intense seed pulse which acts to photoionize the gas as the seed is being amplified. Behind the ionization front formed at the leading edge of the seed pulse, Raman backscattering of the pump into the amplifying seed pulse occurs as in the preformed plasma case. Since the low intensity pump propagates only through the precursor gas, this scheme has the advantage of avoiding any possible premature Raman backscatter of the pump from thermal plasma fluctuations prior to its intended interaction with the seed, an effect which could degrade or entirely disrupt the amplification process [3]. The added benefit of suppressing the formation of nonlinear precursors to the amplifying seed, which could also interrupt amplification, has also been shown in the presence of ionization. Exper-

*Electronic address: dclark@pppl.gov

${ }^{\dagger}$ Electronic address: fisch@pppl.gov imentally, the preparation of the amplifying medium (in this case merely a gas of a specified uniform density) may be far simpler than preparing a plasma of the appropriate density, temperature, and uniformity needed for amplification in the conventional scheme. Like the conventional amplification scheme, however, ionizing amplification also offers the potential of a more robust amplifier by separating the optical system into two components: one component consisting of one or several pump beams delivering large powers over a wide aperture with low requirements on optical precision, and a second system to deliver a higher precision but lower power and smaller aperture seed pulse to extract and focus the pump energy $[4]$.

However, carrying out the ionization of the plasma simultaneous with amplification introduces its own set of constraints on the amplifier parameters [5]. Primarily, the pump must be kept below the photoionization threshold of the gas $\left(I \sim 10^{12-13} \mathrm{~W} / \mathrm{cm}^{2}\right.$ for hydrogen with $\lambda=0.532 \mu \mathrm{m})$ and the initial seed must be sufficiently intense rapidly to photoionize the gas $\left(I \sim 5 \times 10^{14} \mathrm{~W} / \mathrm{cm}^{2}\right.$ for the same case). Constraints must also be satisfied on the gas density: namely, the gas must be sufficiently tenuous that the damping incurred by the seed from ionization does not exceed its amplification rate from Raman backscattering, while it must be sufficiently dense that the backscatter driven Langmuir wave does not break in the fully ionized plasma. An approximate window of $0.001 \leq n_{n} / n_{c} \leq 0.02$ is then available for ionizing amplification with $\lambda=0.532 \mu \mathrm{m}$ and $I_{\text {pump }} \sim 10^{13} \mathrm{~W} / \mathrm{cm}^{2}$ in hydrogen. Here, $n_{n}$ denotes the number density of neutrals, and $n_{c}$ is the critical density at the pump laser frequency. Only hydrogen and helium working gases are useful for this effect. A detailed discussion of the variety of physical processes which can constrain ionizing amplification can be found in Ref. [6].

Ref. [5] analyzed these constraints and demonstrated ionizing Raman amplification numerically using a reduced, 3-wave description of the laser and electrostatic 
fields. This paper extends this work by using the particlein-cell (PIC) code Zohar [7] to verify these results with the greater detail and fidelity possible in a PIC code. Good agreement is found between the PIC and 3-wave results, and the earlier predictions are validated. Specifically, amplification to unfocused output intensities of $\sim 10^{17} \mathrm{~W} / \mathrm{cm}^{2}$ is shown. The interesting effects of blueshifting of the seed (and pump) by the ionization front and ionization-induced Langmuir wake generation are observed but found not significantly to impact the amplification process. The important saturation of the amplification by forward Raman scattering (FRS) of the seed, the same mechanism as expected in a preformed plasma, is also observed.

This paper is organized as follows. Sec. II presents the simulation methods and results using Zohar - in particular, amplification, seed pulse blue-shifting, wake generation, and saturation. The possibility of using a variety of initial seed pulse shapes is also shown. Sec. III discusses the physics of blue-shifting of the pump by the oncoming ionization front of the seed and its impact on amplifi- cation. Sec. IV extends the one dimensional results of the previous sections by discussing a possible two dimensional instability of the seed pulse ionization front and demonstrating that the narrowing of the ionization layer with amplification of the seed can act to suppress this instability. Sec. V then summarizes and concludes.

\section{SIMULATION RESULTS}

For simulations of Raman amplification in ionizing plasmas, the Zohar code required modification to include an ionization package and to run in a "moving window" mode (i.e., following the amplifying seed pulse). The photoionization process was modeled simply by assigning to each cell a small number of neutral gas or "ghost" particles corresponding to bound electrons and ions. Using the electric field magnitude at the center of each cell, the photoionization rate was calculated for that cell using the instantaneous Keldysh formula [8] [24]

$$
w(|a|) \simeq 4 \Omega_{0}\left(\frac{U_{I}}{U_{H}}\right)^{5 / 2} \frac{a_{H}}{|a|} \exp \left[-\frac{2}{3}\left(\frac{U_{I}}{U_{H}}\right)^{3 / 2} \frac{a_{H}}{|a|}\right] .
$$

where $a \doteq|e| E / m_{e} c \omega$ is the normalized amplitude of the total laser electric field, $U_{I}$ the ionization potential, $U_{H}$ the ionization potential of hydrogen, and $a_{H}$ the normalized amplitude of the hydrogenic electric field. Below, $a_{1}, a_{2}$, and $a_{3}$ are used to refer to the normalized amplitudes of the pump, seed, and Langmuir wave envelopes, respectively. The number of atoms ionized (or electrons created) in each cell for one computational time-step is then a binomially distributed random number between zero and the total number of neutrals in the cell where the probability of any given atom ionizing is simply the product of the Keldysh rate and the simulation time-step [25]. Once the number of electrons ionized is calculated, an equivalent fixed positive background charge is also introduced, the number of neutral atoms is reduced correspondingly, and the liberated electrons are free to move under the influence of the local electromagnetic fields. Attention must also be paid to the fact that electrons could be born at any time within the computational timestep $\Delta t$. To include this effect, electrons were assumed to be born at uniformly distributed random times with $\Delta t$ and then given velocities corresponding to having been accelerated in the local electromagnetic fields for the appropriate fraction of a time step. Without accounting for this effect, an unphysical striation of velocity space would result.

Critical in the study of ionizing Raman amplification (on account of the relatively long $\sim 3-6 \mathrm{~cm}$ length scales involved) is the damping of the electric fields introduced by the ionization process. To account for this damping, for every ionization event the magnitude of the electric field vector in each cell was reduced so that the local electric field energy is decreased by the energy required to free the electron, i.e.,

$$
\Delta\left(\frac{|\mathbf{E}|^{2}}{8 \pi}\right) \Delta x=-U_{I} \Delta N_{e}-\sum_{i=1}^{\Delta N_{e}} \tilde{\varepsilon}_{i}
$$

where $U_{I}$ is the ionization potential, $\Delta N_{e}$ is the number of electrons born in a cell of size $\Delta x$ and $\tilde{\varepsilon}_{i}$ represents the kinetic energy of the electron associated with its birth at some random time within $\Delta t$. The magnetic field is not affected by this process. Subsequent to this direct ionization damping, the current resulting from the electrons accelerating in the electromagnetic field on time scales longer than $\Delta t$ results in the appropriate $\mathbf{E} \cdot \mathbf{J}$ damping of the laser pulses.

Note that in fluid codes, ionization damping has previously been implemented by introducing an "ionization current" $\mathbf{J}_{\text {ion }} \doteq w(|\mathbf{E}|) n_{n} U_{I} \mathbf{E} /|\mathbf{E}|$ with $w(|\mathbf{E}|)$ the ionization rate and $n_{n}$ the number density of neutrals $[9,10]$. A formulation of such a current appropriate for a PIC code, however, could not be found. This is a result of the fact that $\mathbf{J}_{\text {ion }}$ represents the rate of energy dissipation (or power) due to ionization and hence must reflect the time scale of the ionization physics $\Delta t_{\text {ion }} \sim \sqrt{2 m_{e} U_{I}} /|e \mathbf{E}|$ 
implicit in $\left|\mathbf{J}_{\text {ion }}\right| \propto \partial_{t} n_{n} \propto 1 / \Delta t_{\text {ion }}$. Since a PIC code represents the physics only on a much coarser time scale $\Delta t \gg \Delta t_{\text {ion }}$, attempting in a consistent and energy conserving manner to represent this current within the PIC framework results in $\left|\mathbf{J}_{\text {ion }}\right| \propto \Delta N_{e} / \Delta t$ and an extreme and artificial reduction of the damping effect. Within the fluid framework, the current may be formulated as $\left|\mathbf{J}_{\text {ion }}\right| \propto w(|\mathbf{E}|) n_{n}$, i.e., without the difficulties of the ionization of discrete electrons over finite time intervals, and this problem is not encountered.

Again on account of the very long interaction lengths in ionizing amplification, a moving window was necessary: PIC calculations involving the full $\sim 10 \mathrm{~cm}$ ionization length with its $\sim 10^{6}$ cells and $\sim 10^{7}$ particles — even in one dimension - are totally unreasonable given current computing speeds. Shortening the problem to calculating the behavior of the plasma only in the immediate vicinity of the amplifying seed is hence required. Such a window was implemented for Zohar by simply shifting all particles and grid quantities by one grid space every second time step to give a window moving at the speed of light when $\Delta x=2 c \Delta t$. Speeds slightly less than $c$, appropriate for following the seed pulse, are selected by simply not shifting in one out of typically every $200-400$ time steps. For runs of several hundred thousand time steps, care had to be taken in adjusting the window velocity to keep pace with the ionization front which propagates at a speed different from the typical seed pulse group velocity due to group velocity dispersion in the ionizing plasma and the effect of ionization damping. At the trailing edge of the simulation window, field quantities and particles may simply be "discarded" provided charge conservation is maintained. At the advancing edge of the window, a uniform loading of neutral gas "ghost" particles is introduced to represent freshly encountered gas. For the electromagnetic fields, the procedure of extrapolating $B_{z}$ and $E_{x}$ at second order and with this calculating $E_{y}$ using the boundary conditions of Sinz [7] was found to be stable and accurate. For the case of ionizing Raman amplification in one dimension, all other field quantities are zero or ignorable at the advancing boundary.

Note that using a moving window simulation for the case of ionizing amplification does not amount to any simplification of the plasma physics of Raman amplification in contrast to the case of a preformed plasma. While in preformed plasmas, the propagation of the pump across the plasma prior to reaching the seed and its stability to thermal Raman backscatter is a critical plasma physics issue deserving of modeling [3], for ionizing amplification no plasma physics occurs ahead of the ionization front, and hence nothing is omitted by not including this in the PIC modeling.

Fig. 1 shows a comparison of snapshots of the leftpropagating electric field envelope from a simulation run with Zohar using a moving window and a simulation run using the simplified 3-wave approximation described in Ref. [5]. The working gas for this case is hydrogen with $n_{n}=0.001 n_{c}$, the pump (not shown) enters the simula-

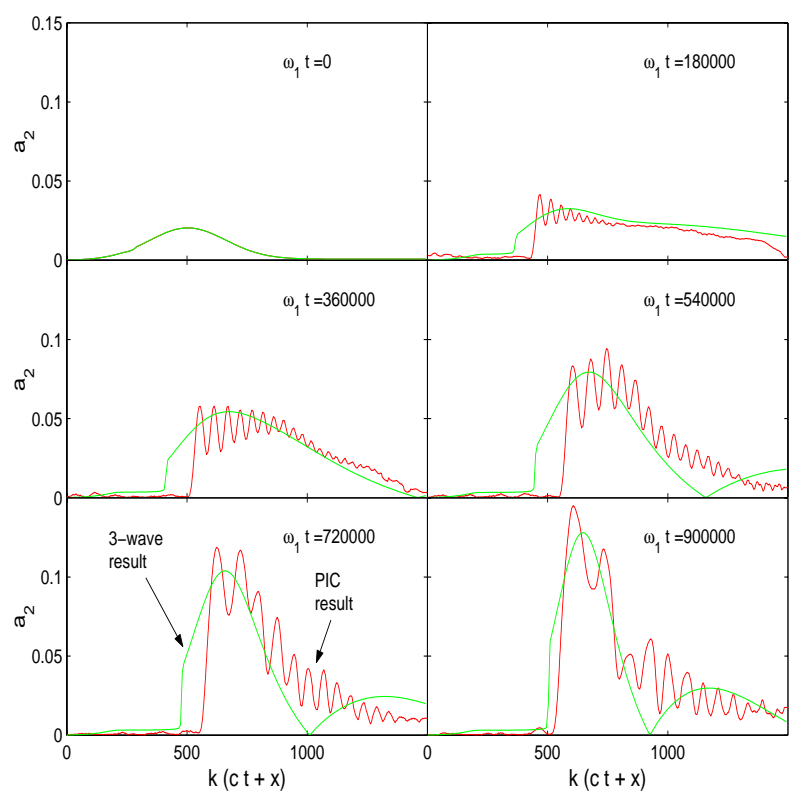

FIG. 1: Snapshots comparing seed pulse envelopes from Zohar (red) and envelope (green) simulations for ionizing amplification in hydrogen with $n_{n}=0.001 n_{c}, a_{1}=0.0015$, $a_{2}(t=0)=0.02$, and $\lambda=0.532 \mu \mathrm{m}$. The pump (not shown) enters from the left. Polarizations are linear.

tion box from the left with an amplitude of $a_{1}=0.0015$, an initial Gaussian seed of amplitude $a_{2}=0.02$ and halfwidth $160 / k_{1}$ is used, and $\lambda=0.532 \mu \mathrm{m}$. Both pulses are linearly polarized.

The 3 -wave simulation (shown in green) displays the typical broadening of the seed during the linear phase of amplification followed by the $\pi$-pulse shape at later times characteristic of the nonlinear phase. The steep ionization front at the leading edge of the seed is evident throughout. The Zohar result (shown in red) echos these trends of initial broadening followed by nonlinear narrowing always accompanied by a steep leading edge. Both simulations reach final intensities of $I \sim 1.2 \times 10^{17} \mathrm{~W} / \mathrm{cm}^{2}$ and pulse widths roughly comparable to the input pulse width. Doubling the number of particles used in the PIC simulation did not significantly alter the results, and convergence of the 3-wave result was verified by repeatedly decreasing the grid spacing. General agreement is seen between the PIC and 3-wave results suggesting that conclusions drawn from the simpler 3 -wave formulation are indeed valid. The strongest distinguishing feature between these simulations is the appearance of a series of slowly growing modulations superimposed on the approximate $\pi$-pulse shape of the PIC results.

Further corroborating the 3 -wave model developed in Ref. [5] are the transverse electron energy distributions measured from the simulation shown in Fig. 2 for $\omega_{1} t=$ 5,000 and 125,000. By comparing with the theoretical distribution given by Burnett and Corkum [11] and used in Ref. [5] for calculating the ionization damping rate, 


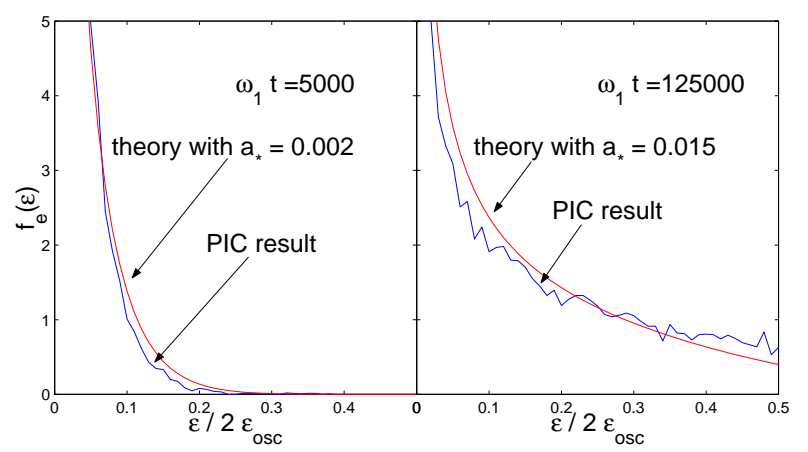

FIG. 2: Comparison of theoretical transverse electron energy distribution (red) with the distribution measured after the passing of the seed pulse from the simulation shown in Fig. 1 (blue) for $\omega_{1} t=5,000$ and 125, 000 .

again good agreement is found. Initially, the measured electron energy distribution shows best agreement with theoretical expectations assuming the majority of electrons are born at a laser field strength of $a_{2} \simeq 0.002$. As should be expected for the early stages of amplification when the seed pulse slope is gentle, the typical birth amplitude corresponds closely to the hydrogen ionization threshold pictured in Fig. 1 of Ref. [5]. At later times, when the seed pulse has steepened due to ionization damping, the characteristic field at which the majority of electrons is born should be given by the estimates of Ref. [5]. As can be seen for $\omega_{1} t=125,000$, using this estimated characteristic amplitude of $a_{*} \simeq 0.015$, the measured electron energy spectrum again agrees closely with the theoretical spectrum.

An insight into the origin of the seed pulse modulations in Fig. 1 is gained by inspecting the $k_{x}$ spectra shown in Fig. 3. The power spectrum of right-propagating energy (i.e., in the direction of the pump) is shown in blue, the spectrum of left-propagating energy (in the direction of the seed) is shown in green, and the spectrum of the longitudinal electric field (i.e., the Langmuir wave) is shown in red. Even for the relatively early time $\omega_{1} t=2.4 \times 10^{5}$, a substantial blue-shifted wing is evident in the leftpropagating spectrum which continues to broaden and grow in amplitude until $\omega_{1} t \sim 7 \times 10^{5}$. This up-shift in $k_{x}$ is the well-known blue-shift expected for an ionizing laser pulse resulting from the continuous co-propagation of the laser field with the density inhomogeneity of the ionization front $[9,12]$. Though not resolved due to the low temporal sampling rate used, a blue-shift in frequency can also be expected due to the ionization front. Also evident is the driven Langmuir wave at $k \simeq 2.0 \omega_{1} / c$ and a broadening of the peak in the left-propagating spectrum which is consonant with the spatial narrowing of the amplifying seed pulse. The growing signal in the longitudinal electric field at very small $k$ 's will be discussed below.

The result of separating the blue-shifted and remaining "red" component of the left-propagating spectrum

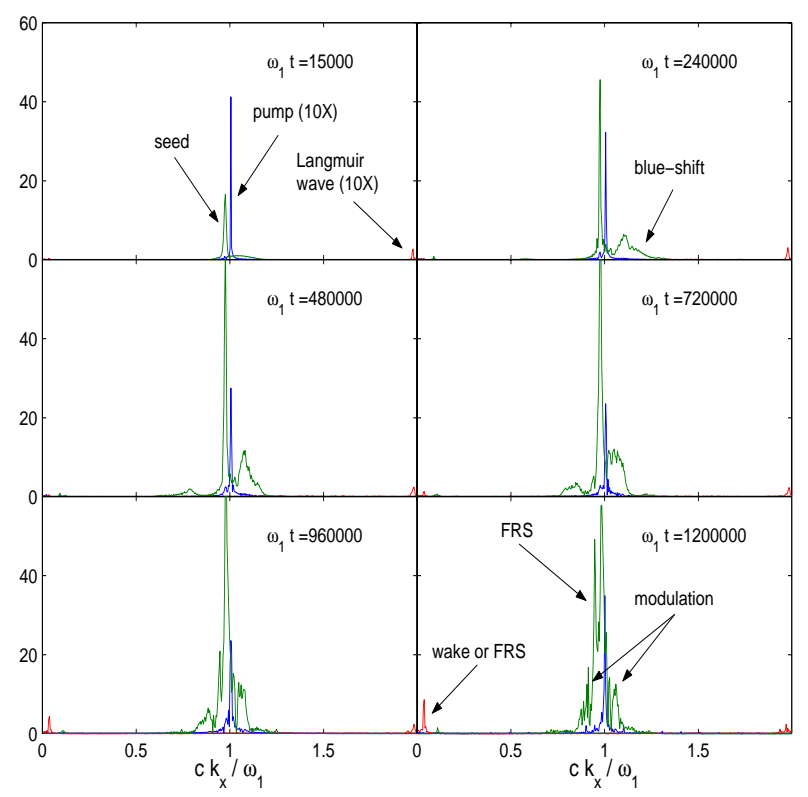

FIG. 3: Snapshots of the $k_{x}$ spectrum from the Zohar simulation shown in Fig. 1. The right-propagating power spectrum is shown in blue, the left-propagating spectrum in green, and the spectrum of the longitudinal electric field is shown in red.

is shown in Fig. 4. With the blue-shifted component fil-

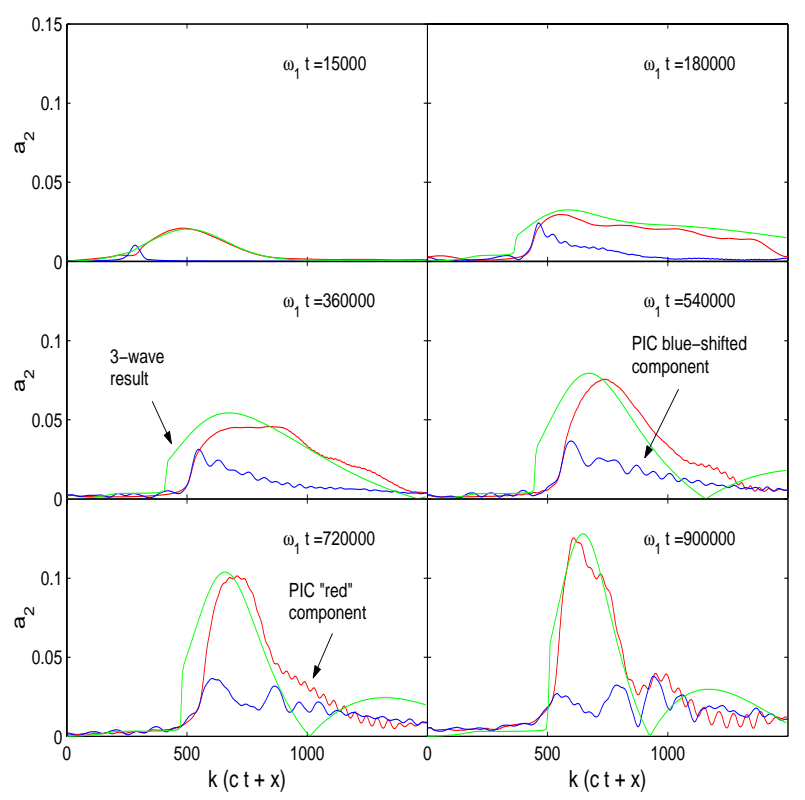

FIG. 4: Snapshots of the filtered seed pulse envelope from the Zohar simulation shown in Fig. 1. The blue-shifted component of the left-propagating field is shown in blue, and the unshifted "red" component is shown in red. For comparison, the result of the 3 -wave simulation is repeated in green.

tered from the signal, the agreement between 3-wave and PIC simulations is seen to be quite close. The agreement confirms both the origin of the seed modulations in the 
ionization-induced blue-shift and again the validity of the 3 -wave model in describing ionizing amplification. Note, however, that a "notching" appears in the peak of the PIC result for $\omega_{1} t \sim 9 \times 10^{5}$.

Running the simulation further, as shown in Fig. 5 without filtering, shows this notch growing into a strong modulation of the seed. The $\pi$-pulse is no longer main-

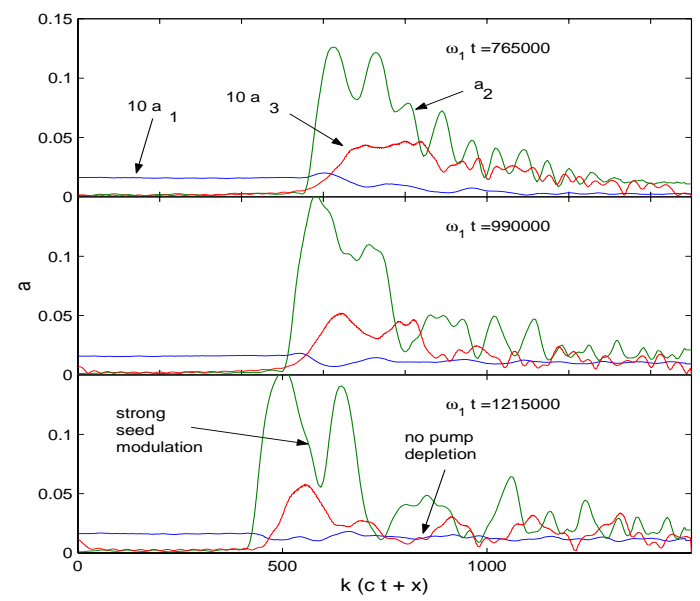

FIG. 5: Snapshots of the late time evolution of the Zohar simulation shown in Fig. 1. The seed envelope is shown in green, and the pump and Langmuir wave envelopes (both multiplied by $10 \times$ for clarity) are shown in blue and red, respectively.

tained, the pump is no longer depleted, and saturation of the amplifying effect is evident. The strong spike in the left-propagating spectrum (Fig. 3) down-shifted by approximately $\omega_{p e} / c$ from the main peak for $\omega_{1} t \geq 9.6 \times 10^{5}$ suggest this to be forward Raman scattering (FRS) of the amplifying seed. That the time of appearance of this signal is approximately the linear growth time for directly forward (i.e., one dimensional) Raman scattering of the seed, $\omega_{1} / \gamma_{\mathrm{FRS}} \sim\left(\omega_{1} / \omega_{p e}\right)^{2} / a_{1} \sim 6.7 \times 10^{5}$, corroborates this. Simultaneously, the broader sidebands near $k^{\prime} \simeq k_{2}\left(1 \pm a_{2}\right) \simeq k_{2}(1 \pm 0.1)$ are the characteristic signature of the modulational instability of the seed with the growth time $\omega_{1} / \gamma_{\text {mod }} \sim\left(\omega_{1} / \omega_{p e}\right) / a_{1}^{4 / 3} \sim 5.1 \times 10^{5}$ [13]. A coupling between the ionization-induced blueshift and the later forward Raman scattering and modulational instabilities is also possible. Note that the observation of saturation of ionizing amplification by FRS and modulation (the same saturation mechanisms expected for Raman amplification in preformed plasmas [1, 14]) confirms that output intensities comparable to those expected for the conventional Raman amplification scheme can be achieved by the ionizing scheme.

In Fig. 6 are shown snapshots of the complete longitudinal electric field. Initially, up to $\omega_{1} t=4.4 \times 10^{5}$, the resonant Langmuir wave takes on the envelope shape expected for 3 -wave coupling and $\pi$-pulse-type behavior behind the ionization front. After $\omega_{1} t=6.5 \times 10^{5}$, however, a Langmuir wake begins to form due to the discontinuity at the ionization front $[15,16]$ and by $\omega t=1.2 \times 10^{6}$ has

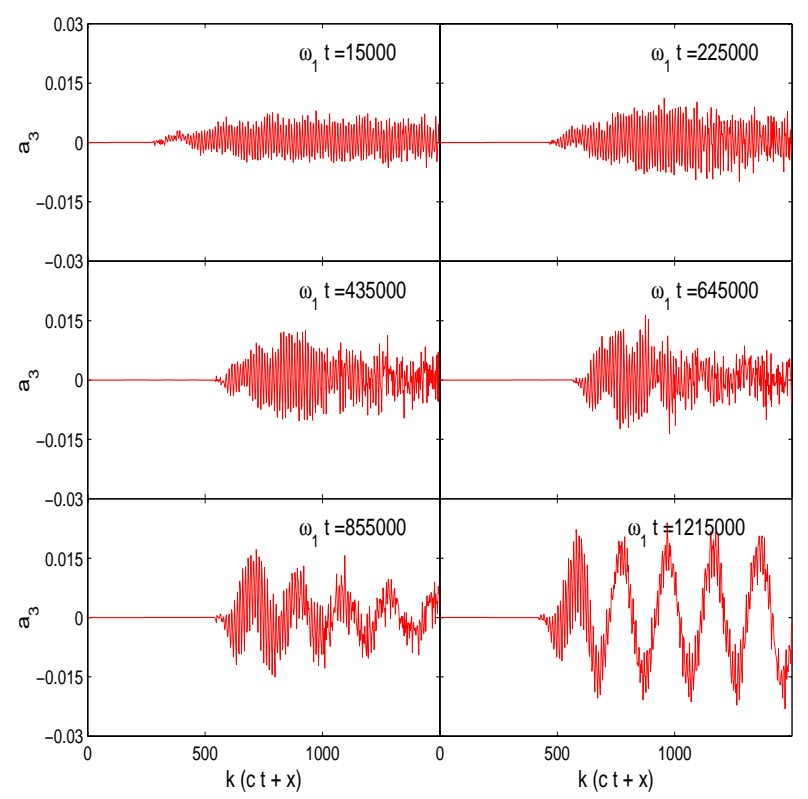

FIG. 6: Snapshots of the complete (i.e., not enveloped) longitudinal electric field from the Zohar simulation shown in Fig. 1.

strongly suppressed the component of the Langmuir wave field resonant for Raman backscattering. Fortunately from the perspective of amplification, this suppression occurs only at times comparable to or after the development of FRS or modulational instabilities and the net amplification of the seed is not then degraded. Wake formation is evident in the longitudinal $k_{x}$ spectrum (Fig. 3) as a growing signal at $k_{x} \simeq \omega_{p e} / c \simeq 0.032 \omega_{1} / c$. Note, however, that the spectral location of this Langmuir wave is effectively indistinguishable from the Langmuir wave that would be driven by FRS of the seed pulse, also at $k_{x} \simeq \omega_{p e} / c$. In effect these instabilities are equivalent.

Repeating the simulation shown in Fig. 1 but increasing the density up to $n_{n}=0.003 n_{c}$ yielded similar amplification results. A density of $n_{n}=0.005 n_{c}$ appeared to be the threshold above which such an initial seed would be extinguished due to ionization damping before amplifying. Likewise, simulations with $\lambda=1.064 \mu \mathrm{m}$ and densities up to $n_{n}=0.003 n_{c}$ showed successful amplification. Notably shorter wavelength ionization-induced modulations appeared on the seed pulses for this longer pump wavelength than those visible in Fig. 1, as was also the case for the higher density simulations.

Initial seed pulse shapes other than the Gaussian shown in Fig. 1 may prove more optimal for ionizing amplification. In Fig. 7 is shown the amplification of a trapezoidal initial seed of peak amplitude $a_{2}=0.007$, i.e., just above the threshold for rapid photoionization with linear polarization. Again, the working gas is hydrogen with $n_{n}=0.001 n_{c}, a_{1}=0.0015$, and $\lambda=0.532 \mu \mathrm{m}$. Despite its relatively low amplitude, the large integrated energy of this longer seed enables it to withstand the photoionization damping for a sufficient length of time 


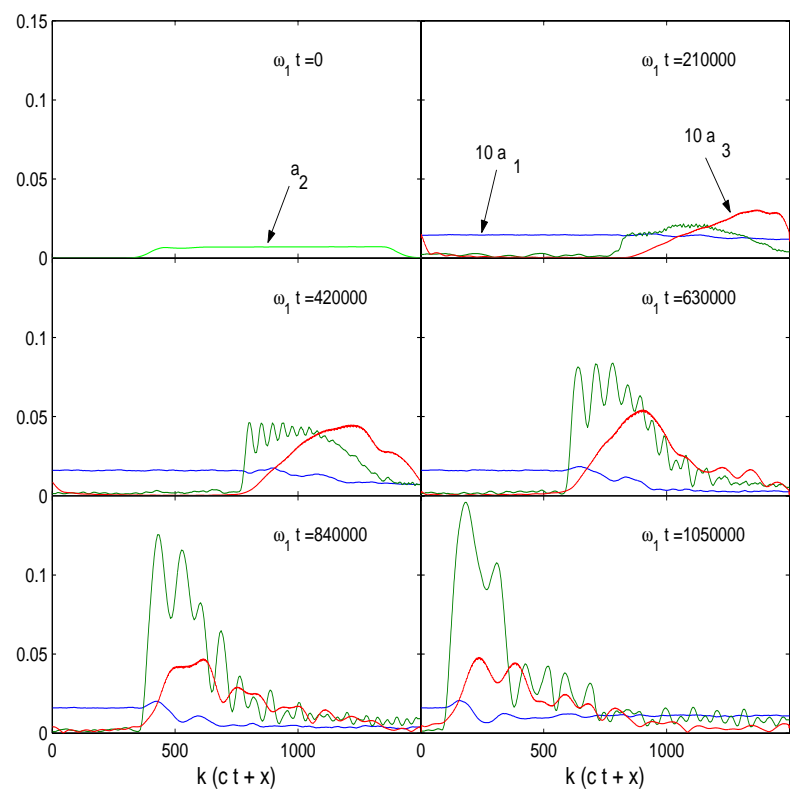

FIG. 7: Snapshots from a Zohar simulation for ionizing amplification in hydrogen with $n_{n}=0.001 n_{c}, a_{1}=0.0015$, $a_{2}(t=0)=0.007$, and $\lambda=0.532 \mu \mathrm{m}$. In this case the seed pulse is initially trapezoidal in shape instead of Gaussian. The seed envelope is shown in green, and the pump and Langmuir wave envelopes (again, both multiplied by $10 \times$ for clarity) are shown in blue and red, respectively. Polarizations are linear.

until the nonlinear phase of Raman amplification has replenished the leading edge of the seed and a robust, amplifying $\pi$-pulse has formed. A net amplification by a factor of $\sim 400$ in intensity to $I \sim 1.2 \times 10^{17} \mathrm{~W} / \mathrm{cm}^{2}$ is then achieved. As shown in Ref. [5], the initial seed pulse shape required to obtain the amplifying and contracting $\pi$-pulse shape is much less stringently constrained in the case of ionizing amplification than it is for amplification in a preformed plasma. While for Gaussian seeds a minimum pulse half-width of approximately $160 / k_{1}$ and initial amplitude $a_{2}(t=0)=0.01$ were found necessary in the simulations for amplification to occur, a great variety of elongated, lower amplitude pulses could be workable for amplification.

\section{BLUE-SHIFTING OF THE PUMP}

In addition to the blue-shift experience by the seed pulse in its co-propagation with the ionization front, a blue-shift of the pump pulse also occurs as it propagates through the oncoming ionization front in an ionizing amplifier. The degree of shift for this process may be calculated by considering (similar to the argument of Ref. [12]) the propagation of an electromagnetic wave through a moving linear electron density ramp

$$
n_{e}(x, t)=\left\{\begin{array}{cc}
0 & x+c t<0 \\
\left(n_{\max } / \Delta\right)(x+c t) & , 0<x+c t<\Delta \\
n_{\max } & , \quad \Delta<x+c t
\end{array}\right.
$$

which approximates the density profile generated by ionization at the leading edge of the intense seed pulse. Here $\Delta$ is the width of the ionization layer determined by the properties of the ionizing seed pulse. When damping is neglected, the electric field amplitude of the pump satisfies the equation

$$
\left(\partial_{t}^{2}-c^{2} \partial_{x}^{2}\right) E=-\omega_{p e, \max }^{2} \frac{n_{e}}{n_{\max }} E
$$

where $n_{e}$ is the local electron number density, and $n_{\max }$ is the number density when the plasma is fully ionized. In terms of the usual moving coordinate $\xi \doteq x+c t$, on the domain $0<\xi<\Delta$, this becomes

$$
\left(\partial_{t}^{2}-2 c \partial_{\xi t}\right) E=-\omega_{p e, \max }^{2} \frac{\xi}{\Delta} E
$$

where the right-moving wave boundary condition at $\xi=$ 0 requires

$$
\begin{aligned}
E(\xi=0, t) & =\left.E_{0} \exp \left[i \omega_{0}(t-x / c)\right]\right|_{x+c t=0} \\
& =E_{0} \exp \left(2 i \omega_{0} t\right) .
\end{aligned}
$$

Decomposing the electric field into an envelope and a rapidly oscillating phase, $E(\xi, t)=\tilde{E}(\xi) \exp \left(2 i \omega_{0} t\right)$ with $\tilde{E}(0)=E_{0}$, then Eq. (2) takes the form

$$
\left(-4 \omega_{0}^{2}+4 i c \omega_{0} \partial_{\xi}\right) \tilde{E}=-\omega_{p e, \max }^{2} \frac{\xi}{\Delta} \tilde{E}
$$

with the straightforward solution

$$
\tilde{E}=E_{0} \exp \left[i \frac{\omega_{0}}{c} \xi\left(\frac{n_{\max }}{8 n_{c}} \frac{\xi}{\Delta}-1\right)\right] .
$$

Returning to the physical field $E(\xi, t)$ and using the definitions of frequency and wave number leads to

$$
\begin{aligned}
\omega & \equiv \frac{\partial}{\partial t} \operatorname{Im} \ln \left(E / E_{0}\right) \\
& =\omega_{0}\left(1+\frac{n_{\max }}{4 n_{c}} \frac{x+c t}{\Delta}\right)
\end{aligned}
$$

and

$$
\begin{aligned}
k & \equiv-\frac{\partial}{\partial x} \operatorname{Im} \ln \left(E / E_{0}\right) \\
& =\frac{\omega_{0}}{c}\left(1-\frac{n_{\max }}{4 n_{c}} \frac{x+c t}{\Delta}\right) .
\end{aligned}
$$

That is, upon exiting the ionization layer $(\xi=\Delta)$, a frequency blue-shift of $\Delta \omega=\omega_{0}\left(n_{\max } / 4 n_{c}\right)$ is achieved. The down-shift in $k$ (as opposed to up-shift in $\omega$ ) should be expected given that the pump is propagating into a 
region of higher $n_{e}$, and hence higher phase velocity, resulting in a stretching of the pulse.

An alternate derivation of this result may be found by following the procedure of Ref. [17] which considered the transmission and reflection of an electromagnetic wave from a sharp (i.e., $\Delta \rightarrow 0$ ) moving discontinuity in the electron density of a plasma, as at an ionization or recombination front. In the frame traveling with the front, the discontinuity is stationary and hence the frequencies of the incident, transmitted, and reflected waves must be equal, $\omega_{i}=\omega_{t}=\omega_{r} \equiv \omega$. Taking the incident wave to impinge on the discontinuity from the left from vacuum, the corresponding wavenumbers are

$$
k_{i}=-k_{r}=\frac{\omega}{c} \quad k_{t}=\frac{\omega}{c} \sqrt{1-\frac{\omega_{p e}^{2}}{\omega^{2}}} .
$$

Lorentz transforming to the frame in which the discontinuity travels with velocity $c \beta$ to the left (the "lab" or primed frame) [18], the incident and transmitted frequencies become

$$
\begin{aligned}
& \omega_{i}^{\prime}=\gamma\left(\omega-c \beta k_{i}\right)=\omega \gamma(1-\beta) \\
& \omega_{t}^{\prime}=\gamma\left(\omega-c \beta k_{t}\right)=\omega \gamma\left(1-\beta \sqrt{1-\frac{\omega_{p e}^{2}}{\omega^{2}}}\right)
\end{aligned}
$$

and the wavenumbers are

$$
\begin{aligned}
& k_{i}^{\prime}=\gamma\left(k_{i}-\beta \omega / c\right)=\gamma \frac{\omega}{c}(1-\beta) \\
& k_{t}^{\prime}=\gamma\left(k_{t}-\beta \omega / c\right)=\gamma \frac{\omega}{c}\left(\sqrt{1-\frac{\omega_{p e}^{2}}{\omega^{2}}}-\beta\right)
\end{aligned}
$$

where $\gamma$ is the relativistic factor. It should be noted that $\omega_{p e}^{2}=\omega^{2}+c^{2} k^{2}$ is a Lorentz scalar and hence $\omega_{p e}^{\prime}=$ $\omega_{p e}$. Denoting the incident frequency in the stationary frame as $\omega_{0} \equiv \omega_{i}^{\prime}$ (as in the preceding calculation), the frequency in the moving frame is given by

$$
\omega=\omega_{0} / \gamma(1-\beta)
$$

and

$$
\frac{\omega_{p e}^{2}}{\omega^{2}}=\frac{\omega_{p e}^{2}}{\omega_{0}^{2}} \gamma^{2}(1-\beta)^{2} \equiv \frac{n_{e}}{n_{c}} \frac{1-\beta}{1+\beta} .
$$

The frequency and wavenumber shifts are then

$$
\begin{aligned}
\omega_{t}^{\prime} & =\frac{\omega_{i}^{\prime}}{1-\beta}\left(1-\beta \sqrt{1-\frac{n_{e}}{n_{c}} \frac{1-\beta}{1+\beta}}\right) \\
& \rightarrow \omega_{0}\left(1+\frac{n_{e}}{4 n_{c}}\right), \beta \rightarrow 1
\end{aligned}
$$

and

$$
\begin{aligned}
k_{t}^{\prime} & =\frac{k_{i}^{\prime}}{1-\beta}\left(\sqrt{1-\frac{n_{e}}{n_{c}} \frac{1-\beta}{1+\beta}}-\beta\right) \\
& \rightarrow \frac{\omega_{0}}{c}\left(1-\frac{n_{e}}{4 n_{c}}\right), \beta \rightarrow 1
\end{aligned}
$$

i.e., exactly the result as before.

In the case of ionizing Raman amplification, for a pump of (vacuum) frequency $\omega_{0}$ launched into a gas which, when ionized, supports $\omega_{p e}=\omega_{0} \sqrt{n_{e} / n_{c}}$, passage through the on-coming ionization front of the seed yields an effective pump frequency of $\omega_{1}=\omega_{0}\left(1+n_{e} / 4 n_{c}\right)$. The Raman resonance condition (neglecting thermal corrections and taking $\beta=1$ ) then requires a seed of frequency

$$
\begin{aligned}
\omega_{2} & =\omega_{1}-\omega_{p e} \\
& =\omega_{0}\left(1+\frac{n_{e}}{4 n_{c}}-\sqrt{\frac{n_{e}}{n_{c}}}\right) .
\end{aligned}
$$

Examples of the magnitude of up-shift in the seed frequency required for various gas densities are given in Table I. For $n_{n} \sim 0.01 n_{c}$, simulations run with Zohar showed noticeably stronger Raman coupling when the effect of this blue-shift was taken into account as compared to selecting the resonance by considering only the peak plasma density. For the more typical $n_{n} \sim 0.001 n_{c}$, however, this effect is negligible.

The frequency up-shift of the pump as it encounters the ionization front in an ionizing Raman amplifier might appear to suggest itself as a means of properly selecting the Raman resonance between the pump and the seed merely by adjusting the plasma density. That is, lasers of the same frequency could be used for both the pump and seed pulses with the pump being "tuned" to the appropriate Raman frequency automatically by it ionization up-shift. Regrettably, satisfying this condition amounts to

$$
\omega_{0}=\omega_{0}\left(1+\frac{n_{e}}{4 n_{c}}-\sqrt{\frac{n_{e}}{n_{c}}}\right)
$$

or the nonsensical requirement $n_{e} / n_{c}=16$.

\section{TRANSVERSE IONIZATION FRONT STABILITY}

The preceding sections have verified the initial predictions of ionizing Raman amplification from 3-wave simulations with the greater detail and rigor of PIC simulations. All of these results, however, have considered only one spatial dimension and neglected the transverse dynamics of the laser pulse. Beyond these one dimensional results, of particular concern is the integrity of the seed pulse to an instability identified in Ref. [19] in which the nonlinear field strength dependence in the Keldysh formula results in a transverse modulation of the front of an ionizing laser pulse. If the growth length of this instability proves to be shorter than that for FRS or modulational instabilities of the seed, then a new (purely two dimensional) mechanism could limit the maximum output intensity for ionizing amplification.

The physical mechanism of this instability can be seen as follows. Any initial transverse perturbation of the laser intensity results in an accompanying perturbation of the 
TABLE I: Example Resonant Seed Frequencies including Pump Blue-Shift

\begin{tabular}{c|c|c}
\hline \hline$n_{e} / n_{c}$ & $\omega_{2} / \omega_{0}$ (shifted) & $\omega_{2} / \omega_{0}$ (unshifted) \\
\hline 0.001 & 0.9686 & 0.9684 \\
0.002 & 0.9558 & 0.9553 \\
0.003 & 0.9460 & 0.9452 \\
0.004 & 0.9378 & 0.9368 \\
0.005 & 0.9305 & 0.9293 \\
0.010 & 0.9025 & 0.9000 \\
\hline \hline
\end{tabular}

electron density following photoionization. The regions of higher electron density can be expected to refract the laser field away from these perturbed regions and lead to lower field intensities and lower ionization rates with a subsequent stabilization of the instability. However, the perturbations imprinted in the electron density, also serve to scatter the laser field. For the appropriate transverse wave vector, this scattered field can act to reinforce the initial field perturbations enhancing the ionization rate and hence amplifying the scattered laser field. An exponential instability thus results.

Neglecting the space and time dependence of the background pulse amplitude $a(x, t)$ and density $n_{e}(x, t)$ so as to derive a dispersion relation, Ref. [19] show that the local gain for this instability is approximately given by

$$
G=\frac{\omega_{p e}}{c} \sqrt{\frac{\kappa}{k_{2}}\left(\xi-\frac{k_{\perp}^{2}}{2 k_{2}^{2}} x\right) x}-\frac{w(|a|)}{c}\left(\xi-\frac{k_{\perp}^{2}}{2 k_{2}^{2}} x\right)
$$

where

$\xi \doteq c t-x \geq \frac{k_{\perp}^{2}}{2 k_{2}^{2}} x \quad$ and $\quad \kappa \doteq \frac{|a|}{2 c}\left(1-\frac{n_{e}(x)}{n_{\max }}\right) \frac{\partial w(|a|)}{\partial|a|}$.

Noting that, for a fixed point in the pulse frame $(\xi=$ const.) the modulation first grows but eventually decays as the pulse propagates and $x$ increases, the instability is seen to be convective in the pulse frame. Hence, at least linearly and within the approximations above, a finite amount of exponentiation can be expected for any initial modulations at any point within the pulse. Further, since the instability can only develop within a layer of width $\Delta$ where the plasma is being ionized, the global maximum exponentiation of an initial seed modulation for the entire pulse is simply given by the maximum of $G(\xi, x)$ over the domain $0 \leq \xi \leq \Delta$. By the method of Lagrange multipliers, this maximum is found to be

$$
\max _{\xi, x} G(\xi, x)=\frac{w(|a|)}{c}[\alpha \sqrt{\beta(1-\beta)}-(1-\beta)] \Delta
$$

with

$$
\begin{aligned}
& \alpha=\frac{\omega_{p e}}{w(|a|)} \frac{k_{2}}{k_{\perp}} \sqrt{2 \frac{\kappa}{k_{2}}} \\
& \beta=\frac{1}{2\left(1+\alpha^{-2}\right)}\left[1+\sqrt{1-\frac{1}{1+\alpha^{-2}}}\right]^{-1} .
\end{aligned}
$$

Roughly, noting that the gain is maximized for $k_{\perp} \sim$ $\omega_{p e} / c$ and that $\kappa \sim w(|a|) / c$ with $k_{2} \sim \omega / c$, then

$\alpha \sim \sqrt{\frac{2 \omega}{w(|a|)}} \quad$ and $\quad \beta \sim\left\{\begin{array}{cl}\left(1+\alpha^{-1}\right) / 2 & , \alpha \gg 1 \\ \left(1+\alpha^{2}\right)^{-1} & , \alpha \ll 1\end{array}\right.$

so that

$$
\max _{\xi, x} G \sim\left\{\begin{array}{cc}
(\Delta / c) \sqrt{\omega w(|a|) / 2}, & \alpha \gg 1 \\
(\omega / c) \Delta & , \alpha \ll 1
\end{array} .\right.
$$

Hence, the maximum degree of exponentiation anywhere in the pulse is evidently directly controlled by the width of the ionization layer $\Delta$.

An estimate of the ionization layer width $\Delta$ may be found as follows. For a laser pulse propagating in a monoelectronic gas of ionization potential $U_{I}$, the laser pulse envelope obeys the equation

$$
n_{c} \frac{m_{e} c^{2}}{2}\left(\partial_{t}-c \partial_{x}\right)|a|^{2}=-\left(U_{I}+\langle\varepsilon\rangle\right) \partial_{t} n_{e},
$$

while the electron and neutral densities evolve according to

$$
\partial_{t} n_{e}=n_{n} w(|a|) \quad \text { and } \quad \partial_{t} n_{n}=-n_{n} w(|a|) .
$$

Again $w(|a|)$ is the Keldysh tunneling photoionization rate, Eq. (1) [26], and $\langle\varepsilon\rangle$ denotes the oscillation energy acquired by the electrons born in the laser field. The effect of laser pulse dispersion due to the varying plasma density is neglected here.

Defining the more convenient variable $y \doteq \beta^{\prime} / a$ with $\beta^{\prime} \doteq(2 / 3)\left(U_{I} / U_{H}\right)^{3 / 2} a_{H}$ and approximating $\langle\varepsilon\rangle \sim U_{I}$, Eqs. (5) and (6) may be combined into a single equation

$$
0=y_{\xi \zeta}+\left(1-\frac{4}{y}\right) y_{\xi} y_{\zeta}+\frac{1}{\epsilon^{\prime}} y e^{-y} y_{\zeta}
$$

with

$$
\xi \doteq \omega\left(\frac{x}{c}+t\right) \quad \zeta \doteq-\frac{\omega}{c} x \quad \epsilon^{\prime} \doteq \frac{\omega \beta^{\prime}}{w_{0}} \ll 1 .
$$

Here $w_{0} \doteq 4 \sqrt{3 / \pi} \Omega_{0}\left(U_{I} / U_{H}\right)^{7 / 4} \sqrt{a_{H}}$, and subscripts denote partial differentiation. Eq. (7) is to be solved on the (moving) domain $\xi>0$ and $\zeta>0$ with the initial 
condition $a(x, t=0)=a_{\text {init }}(\xi)$ and boundary condition that the laser pulse continually encounters fresh gas at $\xi=0$, i.e., $n_{n}(\xi=0, \zeta)=n_{\max }$.

An approximate solution of Eq. (7) may be found using a boundary layer technique which exploits the smallness of $\epsilon^{\prime}[20]$. From the initial condition $a(x, t=0)=$ $a_{\text {init }}(\xi)$, the lowest order solution in $\epsilon^{\prime}$ is simply

$$
a_{\text {out }}(\xi, \zeta)=a_{\text {init }}(\xi)
$$

i.e., the initial pulse shape, unperturbed by ionization. However, to satisfy the boundary condition at $\xi=0$ requires

$1=\left.\frac{n_{n}}{n_{\max }}\right|_{\xi \rightarrow 0}=-\left.\frac{\left(a^{2}\right)_{\zeta}}{R(a)}\right|_{\xi \rightarrow 0} \quad \Longrightarrow \quad a_{\zeta} \sim-\left.\frac{R(a)}{2 a}\right|_{\xi \rightarrow 0}$

where $R(a) \doteq\left(n_{\max } / n_{c}\right)\left(2 U_{I} / m_{e} c^{2}\right) w(|a|) / \omega$. Since the lowest order solution cannot accommodate this boundary condition, a boundary layer must form at $\xi=0$.

Within the boundary layer, the derivatives in $\xi$ dominate, so that the boundary layer behavior is give by the approximate equation

$$
0=y_{\xi \zeta}+\left(1-\frac{4}{y}\right) y_{\xi} y_{\zeta}
$$

Integrating this equation yields

$$
y_{\text {in }}=F^{-1}\left[f(\xi)+\frac{\epsilon}{2 \epsilon^{\prime}\left(\beta^{\prime}\right)^{2}} \zeta+F\left(\frac{\beta^{\prime}}{a_{\text {init }}(\xi)}\right)\right]
$$

where

$$
F(x) \doteq \int^{x} \frac{e^{t}}{t^{4}} d t \quad \text { and } \quad \epsilon \doteq \frac{n_{\max }}{n_{c}} \frac{2 U_{I}}{m_{e} c^{2}} .
$$

The function $f(\xi)$ must be determined by matching together the inner and outer solutions. The matching $y_{\text {in }}=y_{\text {out }}$ is accomplished automatically along the line $\zeta=-\left(2 \epsilon^{\prime} / \epsilon\right)\left(\beta^{\prime}\right)^{2} f(\xi)$, but $y_{\text {in }, \xi}=y_{\text {out }, \xi}$ can simultaneously be satisfied along that line only for the degenerate case $f(\xi)=0$. Since these solutions cannot then be matched together in the usual manner, a nested boundary layer must form between them representing the steepest part of the ionization front of the laser pulse.

Without treating the details of this nested boundary layer, a simpler and more accurate approximate solution may be found by appealing to energy conservation. Neglecting the nested boundary layer, $y_{\text {in }}$ may be matched directly to $y_{\text {out }}$ by choosing $f(\xi)$ so that $0=f(\xi)+\left(\epsilon / 2 \epsilon^{\prime}\right) \zeta /\left(\beta^{\prime}\right)^{2}$ is the trajectory of the matching layer. Conservation of energy up to this boundary then requires

$$
\epsilon \zeta \simeq \int_{0}^{\xi_{\mathrm{match}}} d \xi a_{\mathrm{init}}^{2}(\xi)
$$

That is, the energy invested in ionizing the gas $n_{\max } U_{I} x \propto$ $\epsilon \zeta$ must approximately balance the energy contained in the pulse up to the point $\xi_{\text {match }}$ at which boundary layer matches to the unperturbed initial pulse shape. With this ansatz, the equation for the matching layer trajectory takes the form

$$
\begin{aligned}
f(\xi) & =-\frac{\epsilon \zeta}{2 \epsilon^{\prime}\left(\beta^{\prime}\right)^{2}} \\
& =-\frac{1}{2 \epsilon^{\prime}\left(\beta^{\prime}\right)^{2}} \int_{0}^{\xi} d \xi a_{\text {init }}^{2}(\xi) .
\end{aligned}
$$

For the typical case of a Gaussian initial seed $a_{\text {init }}=$ $a_{0} \exp \left[-\left(\xi-\xi_{0}\right)^{2} / 2 \sigma^{2}\right]$ and $a_{0} \gg \beta^{\prime}$, then

$$
f(\xi)=-\frac{w_{0}}{2 \omega\left(\beta^{\prime}\right)^{3}} \sigma a_{0}^{2} \frac{\sqrt{\pi}}{2}\left[\operatorname{erf}\left(\frac{\xi-\xi_{0}}{\sigma}\right)+\operatorname{erf}\left(\frac{\xi_{0}}{\sigma}\right)\right] .
$$

It should be emphasized that this approximation is valid only for laser pulses much more intense than the photoionization threshold, i.e., it is not valid during the early stages of amplification when the pulse is only just above threshold, but improves in accuracy as the pulse amplifies. A comparison of this solution and a numerical solution of Eqs. (5) and (6) is shown in Fig. 8 for $\lambda=0.532 \mu \mathrm{m}$ and $a_{0}=0.02$. The agreement between the analytical and numerical results is reasonable.

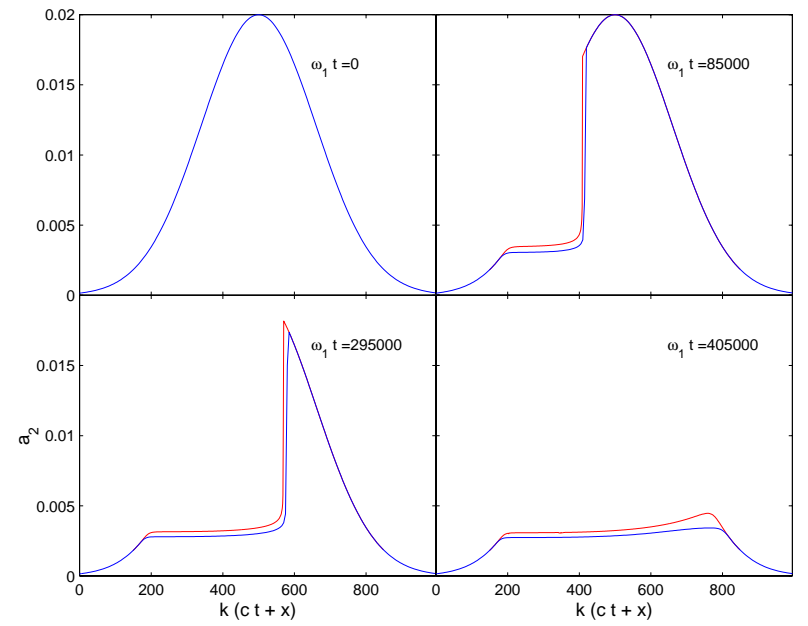

FIG. 8: Comparison of the analytic calculation (red) of the ionization boundary layer shape for an initially Gaussian pulse with the results of numerically integrating Eqs. (5) and (6) (blue) for selected times.

Using the small argument approximation of the error function, $\operatorname{erf}(x) \simeq 2 x / \sqrt{\pi}[21]$, from Eq. (8) the width of the ionization layer is found to scale (again, for $a_{0} \gg \beta^{\prime}$ ) as

$$
\frac{\omega}{c} \Delta \sim 2 \frac{\omega}{w_{0}} \frac{\left(\beta^{\prime}\right)^{3}}{a_{0}^{2}} \propto \frac{U_{I}^{2}}{\omega a_{0}^{2}}
$$

or, noting $n_{c} \propto \omega^{2}$,

$$
\frac{\omega}{c} \Delta \propto \frac{U_{I}^{2}}{a_{0}^{2}} \sqrt{\frac{n_{e}}{n_{c}}}
$$


for fixed frequency. This general scaling of increasing layer width with increasing density and ionization potential and with decreasing frequency has been observed in both 3-wave and PIC simulations. It was also generally observed that $\Delta$ for linear polarizations was greater than for the corresponding case with circular polarization.

This analysis has been for the case of a non-dispersive laser pulse of fixed initial amplitude. Returning to the issue of pulse stability in an ionizing amplifier, Eq. (9) suggests that as the pulse amplifies and $a_{0}$ increases with time, the ionization layer can be expected to narrow $(\Delta \rightarrow 0)$. Hence, from Eq. (4), progressive stabilization of the ionization front can be expected with amplification as the gain is reduced with decreasing $\Delta$. This stabilization due to the narrowness of the ionization layer is also suggested by the work of Ref. [22]. Namely, the instabilities which are observed in the two dimensional simulations of Ref. [22] appear only in the ionization layer of the second ionization state of helium, a layer which (by the scalings of Eq. (9) and that the second ionization potential of helium is approximately twice that of the first ionization state) is much thicker than the first ionization layer. The narrower first ionization layer appears stable to any modulations.

For the case of ionizing amplification, however, the critical issue is whether, for the relevant gas densities and pulse amplitudes, the ionization layer is sufficiently narrow initially or this narrowing happens sufficiently fast adequately to suppress the ionization instability. Further, since the laser pulse amplitude is growing (and hence the ionization layer width is evolving) on a time scale comparable to the instability growth time, neglecting the time dependence of the ionization layer width in assessing the ionization front stability is at best a suspect approximation. While Eq. (9) suggests stabilization by narrowing is possible, the realistic stability of an ionizing Raman amplifier can only be verified using two dimensional simulations.

It should also be noted that several approximations were made in deriving the gain rate Eq. (3). Most notably it was assumed that the pulse shape varies in space and time only slowly compared to $k_{\xi}$ and $k_{\perp}$, i.e., $\partial a / \partial \xi \ll k_{\xi}, k_{\perp}$. As the ionization layer narrows and the pulse front steepens with amplification, this approximation is then gradually invalidated and Eq. (3) may no longer apply. Additionally, the stabilization of the pulse by narrowing of the ionization layer with amplification must compete with the destabilizing effect of the stretching and smoothing of the pulse due to dispersion in the inhomogeneous plasma at the ionization front, an effect completely neglected in Eqs. (5) and (6). This effect will be small for the low density plasmas under consideration but may have a significant cumulative influence over a long interaction length. Again, only realistic, two dimensional simulations can resolve these competing effects.

Regardless, if this ionization front instability proves to be a more stringent limitation on amplification than the modulational or FRS instabilities discussed above, simply initializing the seed with a higher intensity (perhaps from a previous Raman amplifier) and amplifying it over a shorter length could still result in the same output power as if a smaller initial seed were amplified over a longer length in the absence of the instability. Note that Eq. (9) additionally suggests that utilizing shorter wavelength lasers or lower density gases could reduce the ionization layer width and enhance the stability of the ionization front.

On account of the unacceptably long computing times for two dimensional PIC simulations, Zohar proved unsuitable for numerically investigating the development of this transverse ionization instability during amplification. In its place, the faster F3D code [23], which employs an enveloped description of the laser and Langmuir wave fields, was used. Fig. 9 shows the results of such an F3D simulation run to assess the stability of the ionization front during amplification in two dimensions. Plane wave pump and seed pulses were used with hydrogen as the precursor gas. A gas of density $n_{n}=0.01 n_{c}$
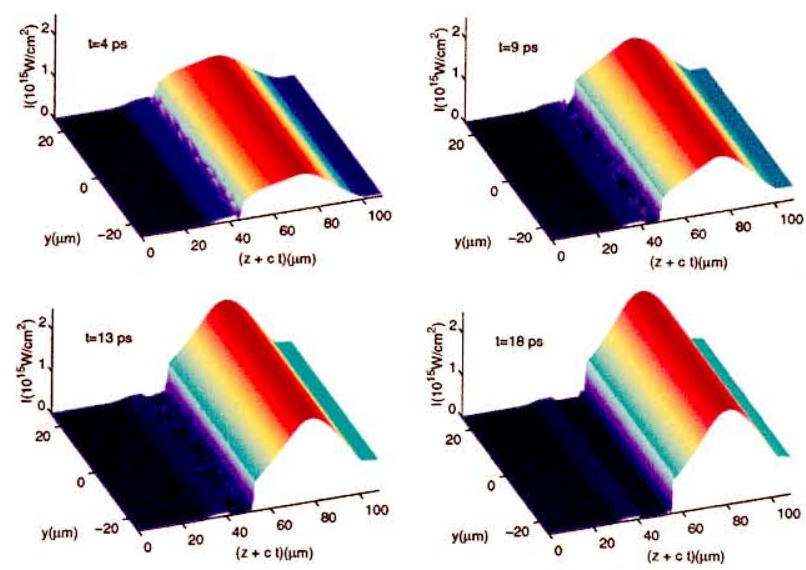

FIG. 9: Snapshots of the seed pulse intensity from a two dimensional simulation of ionizing amplification in hydrogen run with F3D for $a_{1}=0.0015, a_{2}(t=0)=0.007, n_{e}=$ $0.01 n_{c}$, and $\lambda=1.064 \mu \mathrm{m}$.

and wavelength of $\lambda=1.064 \mu \mathrm{m}$ with an initial seed of $a_{2}(t=0)=0.007$ were chosen so that an initially relatively thick ionization layer would develop and maximize the possibility of instability. To trigger the instability, low amplitude random perturbations were added to the initial seed pulse; without this seeding, no transverse modulations developed.

At $t=4 \mathrm{ps}$, the seed perturbations (initially invisible on this scale) are seen to have grown substantially with the dominant mode corresponding to $k_{\perp} \sim k_{2} / 10$. This result agrees with the predictions of Ref. [19] that the linear growth rate is maximized for $k_{\perp} \sim c / \omega_{p e} \sim k_{2} / 10$ for these parameters. At later times $(t=9 \mathrm{ps}$ and $13 \mathrm{ps})$, the modulations develop a highly spiked structure as seen in the simulations of Ref. [22]. More importantly, however, 
ity, low amplitude random perturbations were added to the initial seed pulse; without this seeding, no transverse modulations developed.

At $t=4 \mathrm{ps}$, the seed perturbations (initially invisible on this scale) are seen to have grown substantially with the dominant mode corresponding to $k_{\perp} \sim k_{2} / 10$. This result agrees with the predictions of Ref. [19] that the linear growth rate is maximized for $k_{\perp} \sim c / \omega_{p e} \sim k_{2} / 10$ for these parameters. At later times $(t=9 \mathrm{ps}$ and $13 \mathrm{ps})$, the modulations develop a highly spiked structure as seen in the simulations of Ref. [22]. More importantly, however, in agreement with Eq. (9), as the seed pulse amplifies the ionization layer (visible in the figure as the region in which the perturbations are growing) is seen to narrow. By $t=18 \mathrm{ps}$, the ionization layer has narrowed to the point that the instability is essentially completely suppressed, and at later times the amplification proceeds essentially as in the one dimensional case. Despite having chosen parameters favorable to the development of instability, no catastrophic breakup of the pulse is evident. Further, from similar simulations, the development and saturation of the instability was observed to be independent of the amplitude of the initial seed perturbations provided they are small (less than 10\%) in comparison with the background seed amplitude. From these results, due to the narrowing of the ionization layer with amplification, the instability of Antonsen and Bian does not appear to endanger ionizing amplification.

\section{CONCLUSIONS}

In summary, PIC simulations of ionizing Raman amplification have been presented showing good agreement with previous results based on simplified 3-wave calculations. The peak unfocused output intensity for ionizing amplification was verified to be limited to $I \sim$ $10^{17} \mathrm{~W} / \mathrm{cm}^{2}$ (as in the conventional preformed plasma case) by forward Raman scattering of the amplifying seed. The effects of blue-shifting of the seed in the ionization layer and ionization-induced formation of a Langmuir wake were also observed but found not significantly to affect the seed amplification. Additionally, the blueshifting of the pump laser pulse in propagating through the ionization front of the oncoming seed pulse was shown to have only a small effect for the gas densities of interest in amplification. Finally, it was shown that, due to the narrowing of the ionization layer with amplification of the seed pulse, the transverse ionization front instability identified by Antonsen and Bian should be suppressed in an ionizing Raman amplifier and not disrupt amplification of the seed. This stabilization with amplification was confirmed by two dimensional simulations using the F3D code in which small perturbations of the seed pulse front were initially observed to grow but then be suppressed as the ionization layer narrowed.

\section{ACKNOWLEDGMENTS}

The help of A. B. Langdon in preparing simulations with Zohar and on the general theory and method of PIC simulation is gratefully acknowledged. Assistance from R. L. Berger in preparing simulations with F3D is also gratefully acknowledged. This work was supported by the U.S. Department of Energy Contract No. DEAC02-76-CHO-3073 and the Defense Advanced Research Projects Agency (DARPA).
[1] V. M. Malkin, G. Shvets, and N. J. Fisch, Phys. Rev. Lett. 82(22), 4448 (1999).

[2] V. M. Malkin and N. J. Fisch, Phys. Plasmas 8(10), 4698 (2001).

[3] D. S. Clark and N. J. Fisch, Operating regime for a backward Raman laser amplifier in preformed plasma, to appear, Phys. Plasmas (2003).

[4] N. J. Fisch and V. M. Malkin, Phys. Plasmas 10(5), 2056 (2003).

[5] D. S. Clark and N. J. Fisch, Phys. Plasmas 9(6), 2772 (2002).

[6] D. S. Clark, Ph.D. thesis, Princeton University (2003).

[7] A. B. Langdon and B. F. Lasinski, in Methods in Computational Physics, edited by J. Killeen, R. Alder, S. Fernbach, and M. Rotenberg, Advances in Research and Applications (Academic Press, New York, 1976), vol. 16, p. 327.

[8] L. V. Keldysh, Sov. Phys. JETP 20(5), 1307 (1965).

[9] S. C. Rae and K. Burnett, Phys. Rev. A 46(4), 2077 (1992).

[10] P. Mulser, F. Cornolti, and D. Bauer, Phys. Plasmas 5(12), 4466 (1998).
[11] N. H. Burnett and P. B. Corkum, J. Opt. Soc. Am. B 6(6), 1195 (1989).

[12] E. Esarey, G. Joyce, and P. Sprangle, Phys. Rev. A 44(6), 3908 (1991).

[13] C. Max, J. Arons, and A. B. Langdon, Phys. Rev. Lett. 33(4), 209 (1974).

[14] D. S. Clark and N. J. Fisch, Particle-in-cell simulations of Raman laser amplification in preformed plasmas, in preparation (2003).

[15] W. B. Mori and T. Katsouleas, Phys. Rev. Lett. 69(24), 3495 (1992).

[16] S. Kato, Y. Kishimoto, and J. Koga, Phys. Plasmas 5(1), 292 (1998).

[17] M. Lampe, E. Ott, and J. H. Walker, Phys. Fluids 21(1), 42 (1978).

[18] J. D. Jackson, Classical Electrodynamics (John Wiley \& Sons, New York, 1975), 2nd ed.

[19] T. M. Antonsen, Jr. and Z. Bian, Phys. Rev. Lett. 82(18), 3617 (1999).

[20] E. Zauderer, Partial Differential Equations of Applied Mathematics (Wiley, New York, 1989).

[21] M. Abramowitz and I. A. Stegun, Handbook of Mathe- 
matical Functions (Wiley, New York, 1984).

[22] Z. Bian and T. M. Antonsen, Jr., Phys. Plasmas 8(7), 3182 (2001).

[23] R. L. Berger, C. H. Still, E. A. Williams, and A. B. Langdon, Phys. Plasmas 5(12), 4337 (1998).

[] For the sake of simplicity, only the tunneling ionization rate was used. At low fields, the tunneling ionization approximation is invalid and the (much reduced) ionization rate asymptotes to a multiphoton formula. To model this effect, a simple threshold (corresponding to a Keldysh parameter of $\left.\gamma \doteq \omega \sqrt{2 m_{e} U_{I}} /|e \mathbf{E}|=1\right)$ was introduced below which ionization was neglected entirely.

[] Since only hydrogen and helium are of interest as working gases for ionizing amplification and for simplicity, only the case of a mono-electronic atom of ionization poten- tial $U_{I}$ is considered here. The generalization to multielectron atoms is straightforward but would add little in practical applications.

[] At very low field strengths the photoionization rate obeys a multiphoton formula as opposed to the tunneling formula of Eq. (1). Since this influences the behavior only at the very leading edge of the laser pulse where the field strength is low without significantly effecting the pulse shape at higher fields, this complication is neglected. Also, this calculation has been only for the case of ionization in a circularly polarized laser field. An analysis for the linearly polarized case could be carried out in an analogous manner. 


\section{External Distribution}

Plasma Research Laboratory, Australian National University, Australia

Professor I.R. Jones, Flinders University, Australia

Professor João Canalle, Instituto de Fisica DEQ/IF - UERJ, Brazil

Mr. Gerson O. Ludwig, Instituto Nacional de Pesquisas, Brazil

Dr. P.H. Sakanaka, Instituto Fisica, Brazil

The Librarian, Culham Laboratory, England

Mrs. S.A. Hutchinson, JET Library, England

Professor M.N. Bussac, Ecole Polytechnique, France

Librarian, Max-Planck-Institut für Plasmaphysik, Germany

Jolan Moldvai, Reports Library, MTA KFKI-ATKI, Hungary

Dr. P. Kaw, Institute for Plasma Research, India

Ms. P.J. Pathak, Librarian, Insitute for Plasma Research, India

Ms. Clelia De Palo, Associazione EURATOM-ENEA, Italy

Dr. G. Grosso, Instituto di Fisica del Plasma, Italy

Librarian, Naka Fusion Research Establishment, JAERI, Japan

Library, Plasma Physics Laboratory, Kyoto University, Japan

Research Information Center, National Institute for Fusion Science, Japan

Dr. O. Mitarai, Kyushu Tokai University, Japan

Dr. Jiangang Li, Institute of Plasma Physics, Chinese Academy of Sciences, People's Republic of China

Professor Yuping Huo, School of Physical Science and Technology, People's Republic of China

Library, Academia Sinica, Institute of Plasma Physics, People's Republic of China

Librarian, Institute of Physics, Chinese Academy of Sciences, People's Republic of China

Dr. S. Mirnov, TRINITI, Troitsk, Russian Federation, Russia

Dr. V.S. Strelkov, Kurchatov Institute, Russian Federation, Russia

Professor Peter Lukac, Katedra Fyziky Plazmy MFF UK, Mlynska dolina F-2, Komenskeho Univerzita, SK-842 15 Bratislava, Slovakia

Dr. G.S. Lee, Korea Basic Science Institute, South Korea

Institute for Plasma Research, University of Maryland, USA

Librarian, Fusion Energy Division, Oak Ridge National Laboratory, USA

Librarian, Institute of Fusion Studies, University of Texas, USA

Librarian, Magnetic Fusion Program, Lawrence Livermore National Laboratory, USA

Library, General Atomics, USA

Plasma Physics Group, Fusion Energy Research Program, University of California at San Diego, USA

Plasma Physics Library, Columbia University, USA

Alkesh Punjabi, Center for Fusion Research and Training, Hampton University, USA

Dr. W.M. Stacey, Fusion Research Center, Georgia Institute of Technology, USA

Dr. John Willis, U.S. Department of Energy, Office of Fusion Energy Sciences, USA

Mr. Paul H. Wright, Indianapolis, Indiana, USA 
The Princeton Plasma Physics Laboratory is operated by Princeton University under contract with the U.S. Department of Energy.

\author{
Information Services \\ Princeton Plasma Physics Laboratory \\ P.O. Box 451 \\ Princeton, NJ 08543
}

Phone: 609-243-2750

Fax: 609-243-2751

e-mail: pppl_info@pppl.gov

Internet Address: http://www.pppl.gov 